\title{
A GPS Study on Observing Earthquake Ground Displacement
}

\author{
By Ching-Shun $\mathrm{Ho}^{1)}$ and Chun-Hsiung TSAI ${ }^{2)}$ \\ ${ }^{1)}$ Department of Aeronautics and Astronautics, National Cheng Kung University, Tainan, Taiwan \\ ${ }^{2)}$ Central Weather Bureau, Taipei, Taiwan
}

(Received July 20th, 2009)

\begin{abstract}
Taiwan is located on the boundary between the Eurasian and Philippine Sea Plates. An average motion as high as six cm per year near the boundary has been reported. Earthquake and crustal deformation activities are very high every year. The main purpose of this study is to apply the GPS technique to observe the ground surface motions due to earthquakes. Firstly, the fiducial-site approach was applied to obtain the change between site positions before and after an earthquake. Secondly, the kinematic GPS (KGPS) method is used to obtain time-to-time estimates and to extract the time history of the displacements with a much faster data sampling rate of one second. Two GPS data sets from two earthquake events have been processed. The GPS approaches are capable of detecting surface motions due to earthquakes. The fiducial-site method with daily observation is able to monitor permanent or steady-state site position changes while the KGPS approach with one-second result allows transient response to be estimated.
\end{abstract}

Key Words: Global Positioning System (GPS), Earthquake Displacement, Fiducial-Site Method, Kinematic GPS

\section{Introduction}

Taiwan is located on the boundary between the Eurasian and Philippine Sea Plates. An average motion as high as six $\mathrm{cm}$ per year near the boundary has been reported ${ }^{1)}$. Earthquake and crustal deformation activities are very high every year. An annual average of 1000 sensible earthquakes (Richter magnitude $>3.5$ ) has been detected.

Applying the GPS technique to explore the Earth motion has been quite successful in recent years. Facing such an active surface motion environment, several government agencies in Taiwan have established a number of continuous GPS tracking networks and issued several surveying campaigns to update the local geodetic information to monitor surface changes. For example, the Ministry of Interior (MOI) in Taiwan established a continuous GPS tracking network with eight stations to determine a local datum. In addition, periodic surveying campaigns over 1,000 sites have been performed to check the stability of the local survey control points. The Central Weather Bureau (CWB) of Ministry of Transportation and Communications in Taiwan has set up more than 800 strong motion stations (equipped with three accelerometers) to monitor ground surface motions. To enhance the monitoring function, CWB has been operating a continuous GPS tracking network with more than 140 sites since 2000. Figure 1 is the CWB GPS tracking site distribution that is intended to cover the existing faults.

The fiducial-site concept in processing GPS data is a typical approach to obtain the corresponding crustal deformation $^{2,3)}$. A batch of GPS carrier phase data is processed to yield a relative horizontal position. For a short baseline $(<30 \mathrm{~km})$, the solution accuracy can be of the order of millimeters. The approach allows limited adjustments in site positions and has been successful in static surveying.

Another popular GPS survey technique is referred to as
Kinematic GPS (KGPS) ${ }^{4)}$ and is often used in rapid surveying. The method computes a relative position at each observation time and hence, is capable of monitoring a highly dynamic ground surface motion ${ }^{5,6}$. As a result, two basic ground displacements have been observed. The transient displacement behavior occurs in a very short time period (within seconds) while the steady-state surface change occurs over a period of several minutes ${ }^{6}$.

The main purpose of this study is to apply the GPS technique in observing the ground surface motions due to earthquakes. To accomplish this, both fiducial-site GPS data processing approach and KGPS method have been applied to estimate the ground displacements. The fiducial-site approach is capable of yielding the steady-state or permanent displacements while KGPS method is applied to infer transient position variations. GPS data from Taitung earthquake have been analyzed with these two GPS methods. Data from Hengchun earthquake have been processed with only KGPS approach. To verify the KGPS accuracy, a GPS experiment with $\mathrm{X}$-table facility has been performed prior to the application for the earthquake study.

\section{X-Table Experiment Results}

Experiments with an X-table have been conducted to verify the solution accuracy with the KGPS technique. Shown in Figure 2 is the experimental setup with a GPS antenna on the top of an X-table and an encoder on one side of the table. A typical procedure to execute an experiment is that the operator rotates a handle beside the table to move the antenna according to a designed plan. The encoder records the displacement with micro-meter accuracy that is good enough to assess the KGPS solutions. Since the vertical accuracy from the KGPS solution is poor, the GPS antenna motions were executed in horizontal direction only. 
All of the KGPS experiments were conducted on top of the building of the Institute of Aeronautics and Astronautics (IAA) at NCKU, Tainan, Taiwan. Another GPS receiver was set up about seven meters away from the X-table to be a KGPS reference site (IAA site). Three CWB continuous GPS tracking stations were used as other KGPS reference sites: NCKU (8.3 km in distance), NANK (13 km), and YSAN (22 $\mathrm{km}$, the farthest site from the X-table). As a result, KGPS observations of the moving receiver were computed with respect to the fixed sites of IAA, NCKU, NANK, and YSAN (see site distribution in Figure 3). Accuracy of the GPS observations is expressed by the differences between the encoder readings and the observations at a given time.

Figure 4 shows the GPS antenna moving history of one of $\mathrm{X}$-table experiments that was recorded by the encoder. According to the figure, the antenna was moved in a sequence of steps: moving and staying stationary by four-cm for 30 seconds; by four-cm for 20 seconds; by two-cm for 25 seconds; by two-cm for 35 seconds; moving backward and staying stationary by two-cm for 25 seconds; by two-cm for 20 seconds; and finally by one-cm. At the same time, several GPS receivers to be described below receive and record the GPS observations in every second for the KGPS experiments.

Figure 5 depicts the KGPS results corresponding to the displacements in Figure 4. In the figure, the red line is from KGPS observations of the IAA moving receiver with respect to the fixed site of IAA which is more stable and more accurate than the other three. For example, at $t=70 \mathrm{sec}$. the encoder reading is $-8 \mathrm{~cm}$ (Fig. 4), while the KGPS at IAA also shows $-8 \mathrm{~cm}$ (Fig. 5).

In Figure 5, all of NCKU (green line), NANK (blue line), and YSAN (light blue line) observations show discernible four-cm and two-cm displacements with various accuracy while the one-cm displacement at the end is relatively unobservable by all three. Since the YSAN result is less accurate, the average position during the stationary periods is computed and compared with the encoder readings. The comparison in Table 1 for all displacements indicates that the average KGPS horizontal error is about one-cm.

Table 1. Position Comparison of X-table Experiment.

\begin{tabular}{|c|c|}
\hline Encoder Position $(\mathrm{cm})$ & $\begin{array}{c}\text { YSAN KGPS Position \& } \\
\text { [Standard Deviation] }(\mathrm{cm})\end{array}$ \\
\hline-4 & $-3.5[0.6]$ \\
\hline-8 & $-8[0.3]$ \\
\hline-10 & $-10.5[0.4]$ \\
\hline-12 & $-13[0.6]$ \\
\hline-10 & $-9.5[0.4]$ \\
\hline-8 & $-8[0.6]$ \\
\hline-7 & $-6[0.6]$ \\
\hline
\end{tabular}

\section{Taitung Earthquake Data Processing}

On April 1, 2006, an earthquake measured 6.4 in Richter scale and $7.2 \mathrm{~km}$ in depth struck Taitung City area. It caused a short term power outage for more than 7000 houses. It was the largest earthquake occurred in the area over a three-year span. On the boundary between the Eurasian and Philippine Sea Plates, there is a famous fault called Longitudinal Valley
Fault (LVF) in Taiwan that a crustal deformation of at least five $\mathrm{cm} /$ year has been observed. In addition, there are several CWB GPS continuous tracking sites located along the fault. The Taitung earthquake occurred in the fault area. As a result, more information regarding the crustal motion can be obtained with the GPS data.

There were ten CWB GPS sites close to the epicenter (yellow star in Figure 6) of the Taitung Earthquake. Firstly, this study applied the fiducial-site approach to obtain the change between the site positions before and after the earthquake. Figure 6 shows the horizontal displacements based on this approach. Most of the sites (e.g. LONT, PEIN, or TTUN) near LVF and close to the epicenter have larger changes than the others (e.g. CHUL, CHEN, or DULI) which are away from. The largest displacement of five centimeters occurred on the PEIN site.

A further fiducial-site study with longer time span is performed. Figure 7 shows the daily TTUN-PEIN baseline observations over five months. A gap of one $\mathrm{cm}$ in the north and that of $2.5 \mathrm{~cm}$ in the east (positive sign means moving eastwardly) can be observed in the figure. The changes are close to the results in Figure 6. It indicates that an earthquake may cause a rapid surface change that becomes permanent.

Secondly, the KGPS data processing method is applied to a much smaller data batch of 30 minutes in length and one second in data sampling. Since the KGPS observation is estimated in terms of relative position, relative changes with the fiducial-site approach among these sites are computed. According to Figure 6, the baseline from PEIN to TTUN (TTUN-PEIN) has changed approximately by three centimeters $(1.5 \mathrm{~cm}$ in the north and two $\mathrm{cm}$ in the east). TTUN and LONT sites are found to move in opposite directions, As a result, the TTUN-LONT baseline change of two $\mathrm{cm}$ in the north and two $\mathrm{cm}$ in the east magnifies the relative displacements.

Shown in Figure 8 are the KGPS observations varying with time of the TTUN-PEIN baseline. Jumps in three directions reflect a phenomenon of transient surface motion. The magnitude of the jump phenomenon can be as high as ten centimeters and last only two to five seconds. It indicates the occurrence of the earthquake. After the surface transient response, the response becomes a steady-state relative displacement. If the averages before and after the jump are taken, the differences can be seen as steady-state relative changes. The relative change in the east direction (green line) in Figure 8 is about $3.5 \mathrm{~cm}$ while it is $0.5 \mathrm{~cm}$ in the north (blue line). The changes are slightly different from the steady-state observations in Figure 6 by around one centimeter which is about the error size of the typical KGPS horizontal accuracy of $0.6 \mathrm{~cm}$ in Table 1.

Shown in Figure 9 are the relative displacements of TTUN-LONT baseline. Three jumps indicate the earthquake occurrence at 1354 seconds (true earthquake time is at 1353.4 seconds) that is consistent with the occurrence in Figure 8 of the TTUN-PEIN baseline case. Since TTUN and LONT sites are heading opposite in the east direction, the steady-state relative change in the east (green line) becomes relatively large and is about $2.5 \mathrm{~cm}$. Both the north and east relative changes are around two $\mathrm{cm}$ and are consistent with the 
fiducial-site result

By comparing the observations among the long arc (five months), two-day arc, and short arc (30 minutes), the horizontal changes are consistent in terms of direction and magnitude. It suggests that the major part of a permanent change due to an earthquake may be formed in a very short time span (e.g. less than ten seconds with a Richter magnitude of 6.4).

\section{Hengchun Earthquake Data Processing}

At the end of Year 2006, another large earthquake measured 7.0 in Richter scale and $44.1 \mathrm{~km}$ in depth struck southern Taiwan. There were two main quakes in the earthquake event. According to the published news issued by $\mathrm{CWB}$, the epicenters were more than $30 \mathrm{~km}$ away from Hengchun. Although it was not a shallow one, the damages were still very high. Fortunately, the occurrences of these two main quakes were apart by around six minutes. There were several CWB GPS tracking stations close to the epicenters (see red star in Figure 10 and only the data of CLON and HENC were processed in this study).

Any baseline to LIUC or HENC sites is more than $20 \mathrm{~km}$ in length which may make KGPS technique more challenging. Figure 11 shows the position change of HENC-CLON baseline KGPS result. It is clear that there are two earthquakes (two jumps for each color line in Figure 11) as reported in the news and were 474 seconds apart. The maximum variation in the east direction (green line) of the first jump is around $15 \mathrm{~cm}$. The maximum transient response of the second jump is even larger with $25 \mathrm{~cm}$ in variation. The maximum northern variation (blue line) of the first jump is around $15 \mathrm{~cm}$ while the second one is smaller with $10 \mathrm{~cm}$ in variation.

The steady-state variation in the east direction (green line) shows a step-like pattern so that after an earthquake, the variation becomes a step down. The average differences before and after the earthquakes were computed. The first change in the east is about two centimeters and the second step is three centimeters. The total change is around five centimeters in the east. The steady-state change in the north is relatively small comparing with the change in the east. The result may imply that the larger the transient motion induces the bigger position changes in both steady-state and transient variations.

\section{Conclusions}

This study showed that the GPS technique was capable of measuring the Earth surface displacements caused by earthquakes. It was observed that the displacements could be categorized into two parts: the transient and the steady-state parts. The transient part occurred in a very short time span of several seconds. However, the magnitude could be five times higher than the steady-state value. The results from Taitung earthquake with five-month arc, two-day arc, and 30-minute arc data sets suggested that permanent change due to an earthquake could be formed in a short time span. In addition, the results from Hengchun earthquake confirmed that the transient displacement could be 18 centimeters large while the steady-state one was within four centimeters. In the future, further study by combing the accelerograph and GPS data will be conducted to provide continuous dynamic surface responses of crossing a fault.

\section{Acknowledgments}

This research is supported by the National Science Council of R.O.C. in Taiwan under Grant NSC 97-2221-E-006-098 and by Central Weather Bureau (CWB) of Ministry of Transportation and Communications of R.O.C. in Taiwan under Grant MOTC-CWB-96-E-16.

\section{References}

1) Pezzopane, S. K. and Wesnousky, S. G.: Large Earthquakes and Crustal Deformation Near Taiwan, Journal of Geophysics Research, 94 No. B6, June 10, (1989), pp.7250-7264.

2) Kaniuth, K., Stuber, K., Schmitz-Hubsch, H. and Tremel, H.: Analysis of A Global GPS Network Based on GIG'91 Data, Proceedings of The $6^{\text {th }}$ International Geodetic Symposium on Satellite Positioning, Vol. I, Columbus Ohio, U.S.A., March 17-20, (1992), pp.381-388.

3) Bock,Y., Agnew, D. C., Fang, P., Genrich, J. F., Hager, G. H Herring, T. A., Hdnut, K. W., King, R. W., Larsen, S., Minster, J. B., Stark, K., Wdowinski, S. and Wyatt, F. K.: Detection of Crustal Deformation from the Landers Earthquake Sequence Using Continuous Geodetic Measurements, Nature, 361(1993), pp.337-340.

4) Mader, G.: Kinematic GPS Phase Initialization Using the Ambiguity Function, Proceedings of The $6^{\text {th }}$ International Geodetic Symposium on Satellite Positioning, II Columbus Ohio, U.S.A., March 17-20, (1992), pp.712-719.

5) Chung, J.-K. and Shin, T.-C.: Implications of the Rupture Process from the Displacement Distribution of Strong Ground Motions Recorded during the 21 September 1999 Chi-Chi, Taiwan Earthquake, Terrestrial Atmospheric and Oceanic Sciences, 10 No. 4, December (1999), pp.777-786.

6) Ho, C. S., Lee, C. F., Yu, J. Y. and Tsai, J. H.: Dynamic Surface Response of A Local Area Induced by Chi-Chi Earthquake Using Accelerograph and GPS Solutions, Proceedings of ION National Technical Meeting 2004, San Diego CA, U.S.A., January 26-28, (2004), pp.1005-1010. 


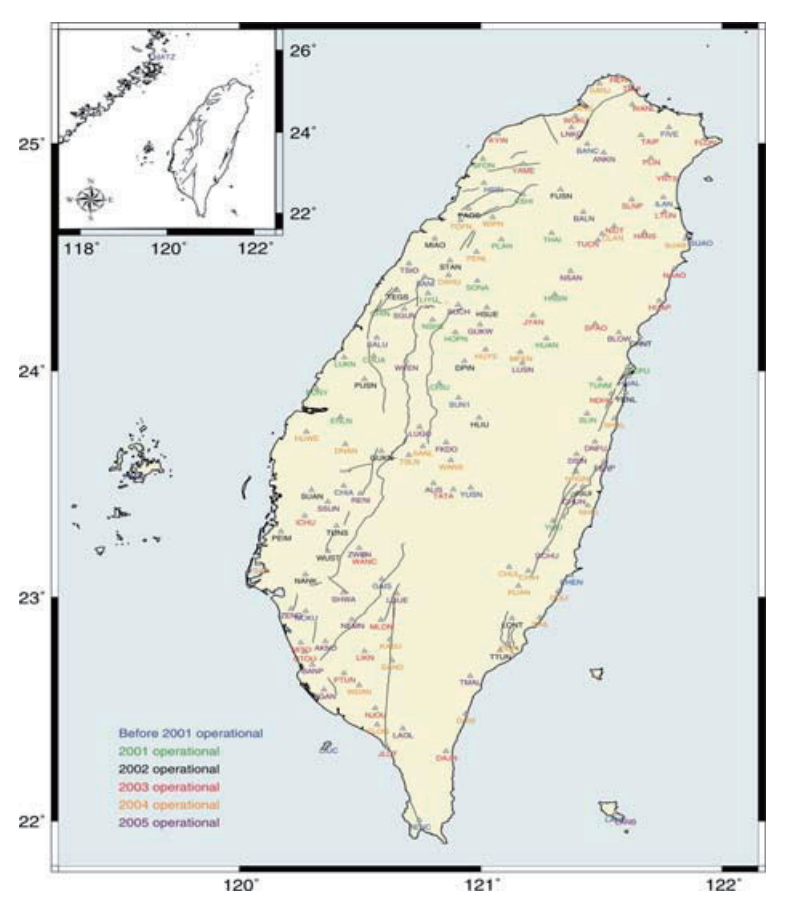

Fig. 1. The distribution of CWB GPS tracking stations.

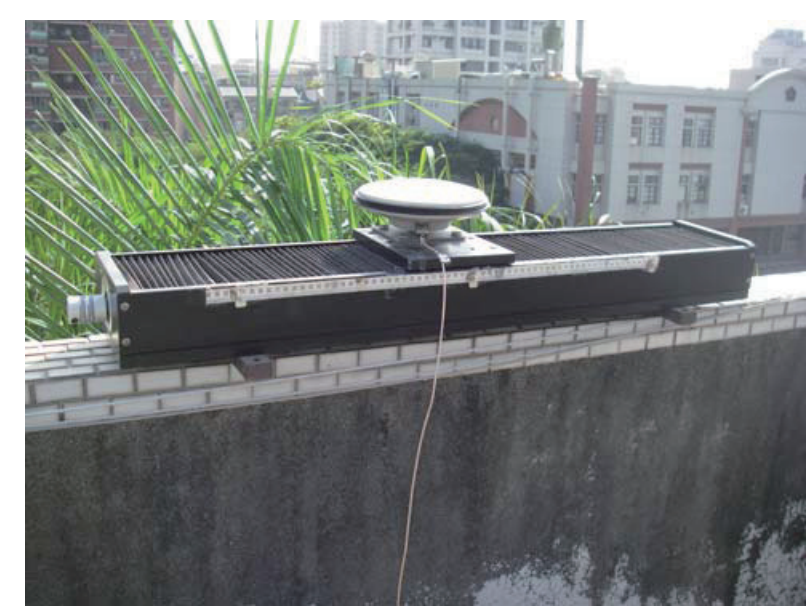

Fig. 2. Experiment setup with a GPS antenna on the top of an X-table.

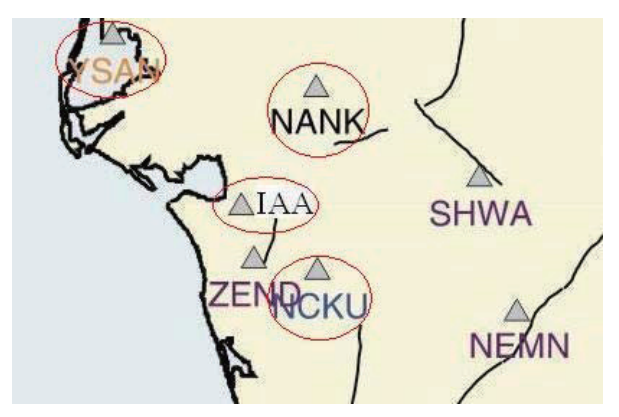

Fig. 3. GPS sites distribution of X-table experiments.

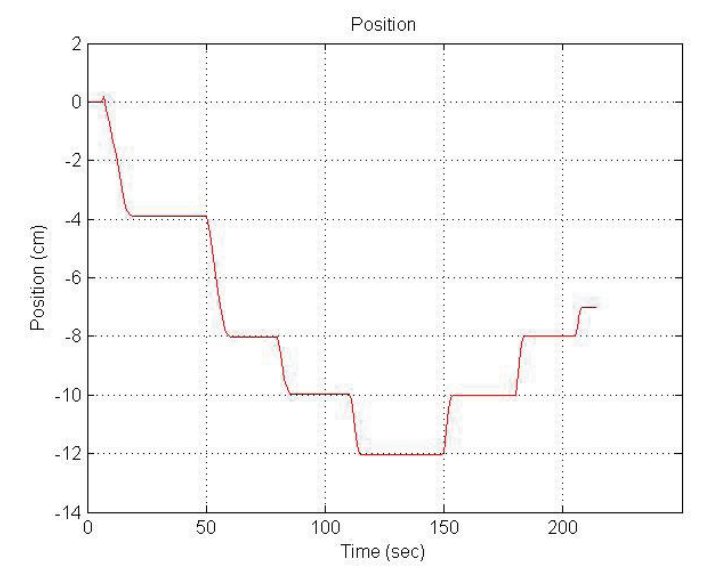

Fig. 4. GPS antenna moving history of one of X-table experiment.

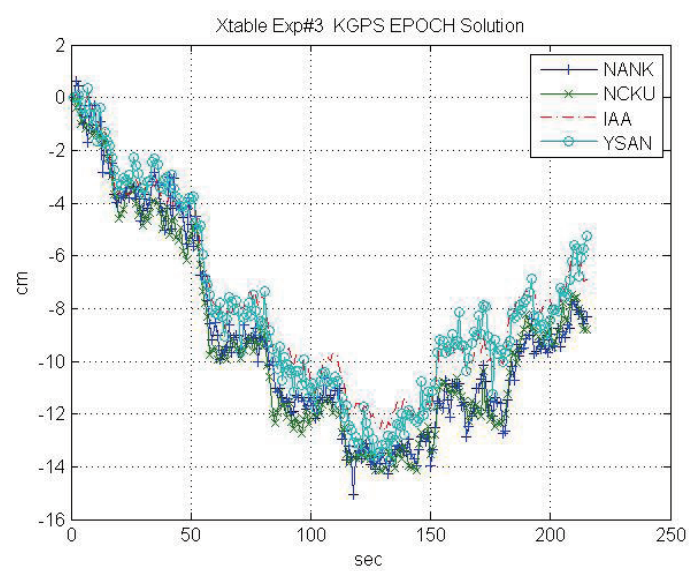

Fig. 5. KGPS results corresponding to the displacements in Figure 4.

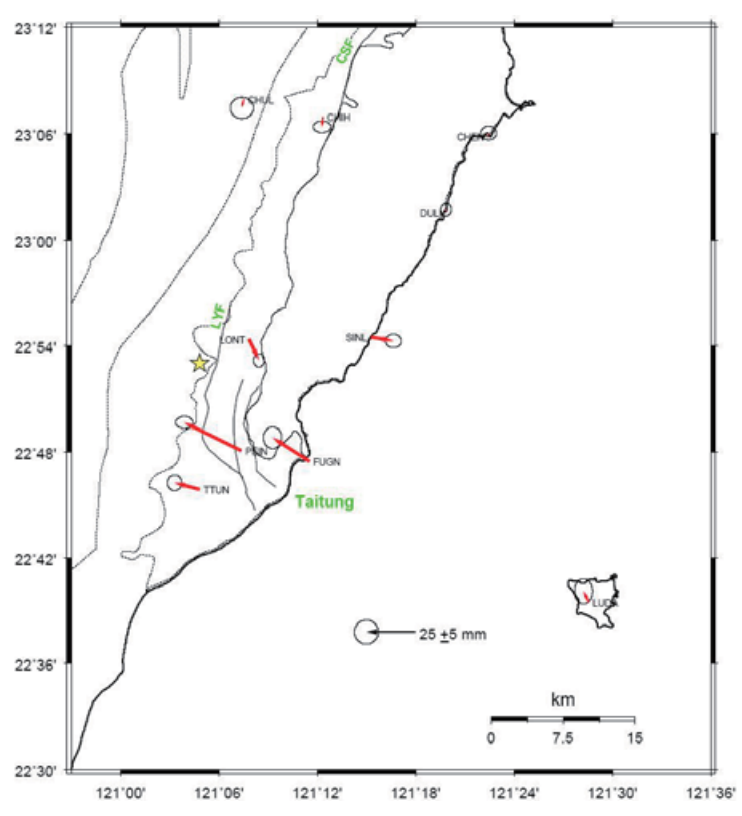

Fig. 6. 2006/04/01 Taitung Earthquake information. 

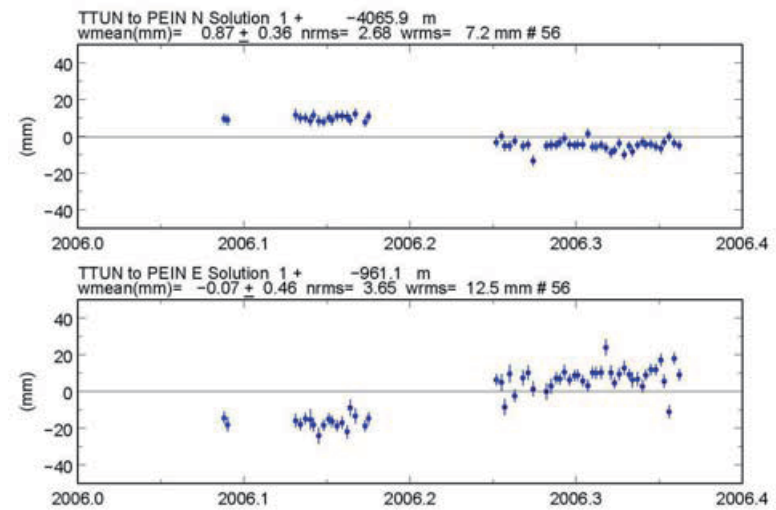

Fig. 7. Taitung Earthquake (at $2006 / 04 / 01$ or 2006.25 year) TTUN-PEIN fiducial-site result.

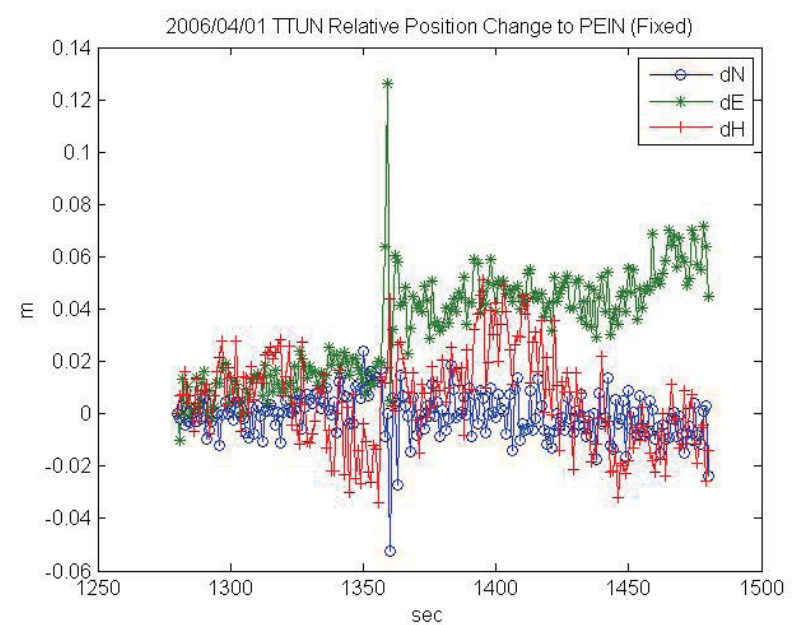

Fig. 8. Taitung Earthquake TTUN-PEIN KGPS result starting from 2006/4/1 9:40 AM.

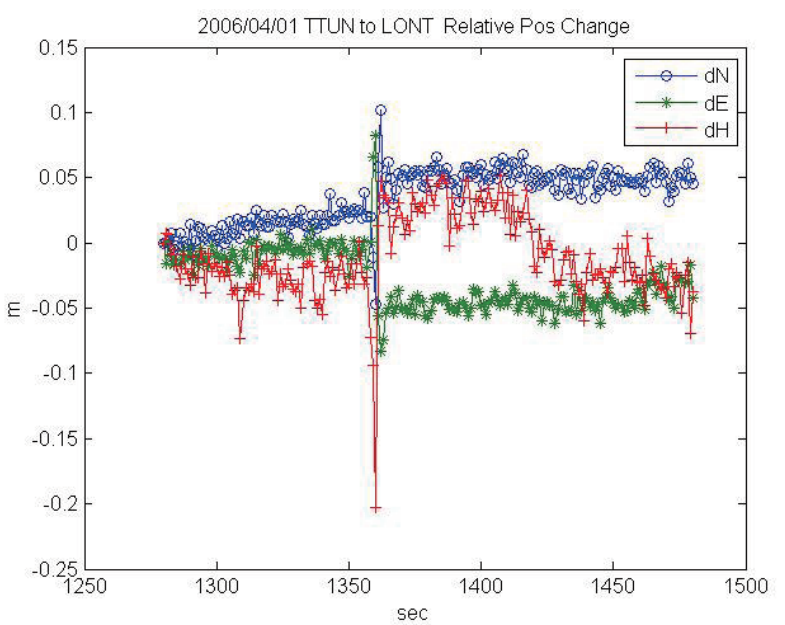

Fig. 9. Taitung Earthquake TTUN-LONT KGPS result starting from 2006/4/1 9:40 AM.

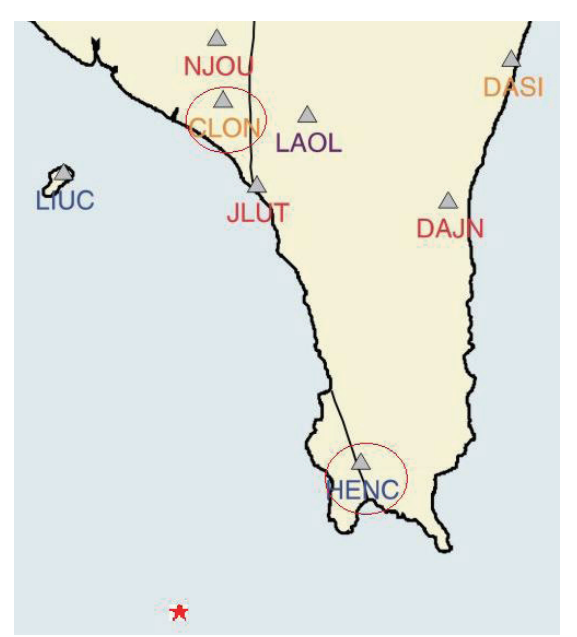

Fig. 10. 2006/12/26 Hengchun Earthquake GPS sites.

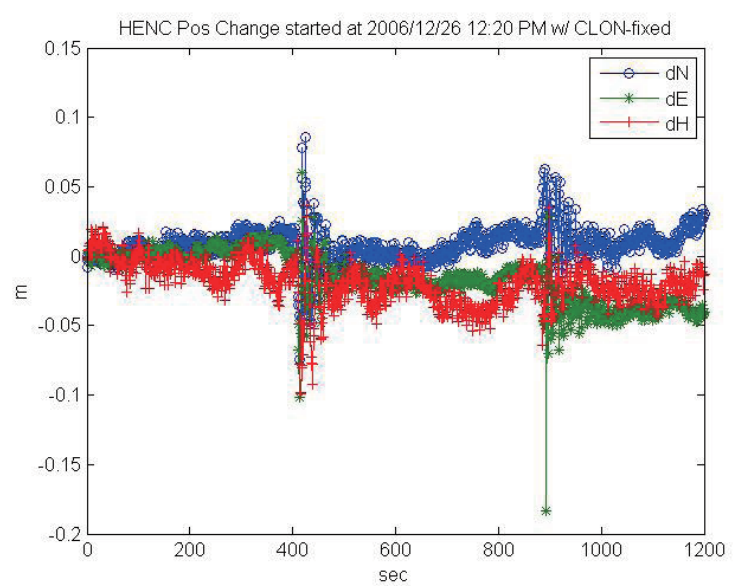

Fig. 11. Hengchun Earthquake HENC-CLON KGPS result. 